\title{
Safety and Feasibility of Autologous Micro-Fragmented Adipose Tissue Injections for the Treatment of Vaginal Atrophy, Vulvovaginal Dystrophy, and Stress Urinary Incontinence: An Observational Case Series
}

\author{
Authors: $\quad$ *Laura Stark, Mira Razzaque, Jeannie Yoon, Mehrnoosh Aref-Adib, \\ Miles Banwell, Shohreh Beski \\ The Regenerative Clinic, London, UK \\ ${ }^{*}$ Correspondence to Is2u16@soton.ac.uk \\ Disclosure: $\quad$ The authors have declared no conflicts of interest. \\ Received: $\quad 13.10 .2019$ \\ Accepted: $\quad 05.12 .2019$ \\ Keywords: $\quad$ Mesenchymal stem cells, stress urinary incontinence (SUI), vaginal atrophy, \\ vulvovaginal dystrophy. \\ Citation: $\quad$ EMJ Urol. 2020;8[1]:29-37.
}

\section{Abstract}

Objective: Vaginal atrophy, vulvovaginal dystrophy, and stress urinary incontinence (SUI), common conditions in women, have detrimental effects on quality of life. Current treatments require ongoing use and are associated with risks, complications, and incomplete resolution of symptoms. The aim of this observational case series was to evaluate the safety and feasibility of autologous micro-fragmented adipose tissue injections for the treatment of vaginal atrophy, vulvovaginal dystrophy, and SUI in women.

Methods: Ten women affected by vaginal atrophy, vulvovaginal dystrophy, and/or SUI were injected into their affected areas with harvested and processed autologous micro-fragmented adipose tissue. Symptoms, diagnoses, previous treatments, and gynaecological surgeries were considered. Outcomes were measured using the Female Sexual Function Index (FSFI), the International Consultation on Incontinence Questionnaire-Urinary Incontinence Short Form (ICIQ-UI SF), the Vulvovaginal Symptoms Questionnaire (VSQ), and the Short-Form 12 (SF-12) health survey. Information gained from discussion and clinical examination at consultations was also used to measure outcomes.

Results: No adverse events or complications were reported during the intraoperative, recovery, postoperative, or follow-up periods. No signs of infection, pain, dysuria, skin irregularities, skin discolouration, discharge, or worsening of symptoms were reported. All 10 women reported an improvement of symptoms within 6-16 months of the planned follow-up period.

Conclusions: Autologous micro-fragmented adipose tissue injections appeared to be safe and feasible and may have a positive role in the treatment of the physical signs and symptoms of vaginal atrophy, vulvovaginal dystrophy, and SUI. 


\section{INTRODUCTION}

Vaginal atrophy, vulvovaginal dystrophy, and stress urinary incontinence (SUI) are conditions that have significant implications for quality of life. They can occur at any time during a woman's life, but are most common in postmenopausal women, with a prevalence of up to $54.0 \%$, $1.7 \%,{ }^{3}$ and $31.0 \%,{ }^{4}$ respectively. Symptoms of vaginal atrophy and vulvovaginal dystrophy include vaginal dryness, decreased lubrication during sexual activity, irritation, itching, dysuria, dyspareunia, and postcoital bleeding. These symptoms, alongside the involuntary urination of SUI, can have a detrimental effect on urogenital health, sexual function, and psychological wellbeing. . $^{5}$

\section{Vaginal Atrophy}

Vaginal atrophy, also known as genitourinary syndrome of the menopause, is a common condition that is often under-diagnosed and under-recognised. The urogenital changes that cause the symptoms of vaginal atrophy occur as a result of a reduction in circulating oestrogen levels and therefore current treatments are targeted at replacing oestrogen through systemic or topical approaches. These treatments have been proven to have a therapeutic effect on the symptoms of vaginal atrophy, but the benefit of one modality over another is still debated. Furthermore, they require ongoing therapy and adherence is poor because of issues related to side effects, convenience, and worries surrounding increased risk of cancer with hormone therapies. ${ }^{9,10}$ Additionally, $<50 \%$ of women using prescribed treatments report satisfaction with the treatment and $10-20 \%$ of women report residual symptoms. ${ }^{2,10}$

\section{Vulvovaginal Dystrophies}

Vulvovaginal dystrophies such as lichen sclerosus, lichen planus, and lichen simplex are chronic inflammatory dermatoses with an unknown aetiology and are associated with an increased risk of the development of vulvar malignancies. ${ }^{11,12}$ First-line treatment is limited to ultrapotent topical corticosteroids, but complete resolution is only reported in approximately $20 \%$ of patients and many experience skin thinning as a side effect.13
Surgery is only indicated in cases of malignancy and postinflammatory sequelae, and for treatments such as photodynamic therapy and cryotherapy, relapse rates are high. ${ }^{14}$

\section{Stress Urinary Incontinence}

SUI describes the involuntary urination upon effort, exertion, sneezing, or coughing. The current nonsurgical therapies for SUI include physiotherapy, intravaginal devices, urethral inserts, and pharmacological treatment. However, these strategies require ongoing treatment and behaviour modification, which may reduce adherence to a regime. Furthermore, surgery to treat SUI can be associated with complications and recurrence of symptoms, and repeat surgery is required in up to $14.5 \%$ of women. ${ }^{15}$

Advancements in regenerative medicine over the last decade have revealed the potential for mesenchymal stem cells (MSC) to be used as a powerful therapeutic tool against tissue damage and degeneration. Not only do MSC possess multilineage potential, but the secretion of trophic factors act in a paracrine fashion to promote an antifibrotic, antiapoptotic, and angiogenic response in the recipient tissues, ultimately producing an anti-inflammatory and immunomodulatory healing effect. ${ }^{16}$ Adipose tissue has been recognised as a reliable source for MSC $^{17,18}$ which can be easily harvested and processed using the Lipogems ${ }^{\circledast}$ system (Lipogems International S.p.A., Milan, Italy), where intraoperative mechanical manipulation provides micro-fragmented adipose tissue in a short period of time. ${ }^{19}$ Furthermore, mechanical manipulation of adipose tissue results in greater preservation of trophic factors compared to traditional enzymatic methods used for harvesting adipose-derived stem cell. ${ }^{20}$

Autologous micro-fragmented adipose tissue injection is well established as a safe and effective treatment and has several clinical applications. ${ }^{21-24}$ The use of autologous adipose tissue in the treatment of vaginal atrophy has provided some promising results, but evidence is limited. ${ }^{25-31}$ Micro-fragmented adipose tissue was used by Fantasia et al. ${ }^{27}$ for the treatment of vaginal atrophy in one woman and by Casarotti et al. ${ }^{28}$ in three women. A significant improvement in symptoms was reported, 
without any complications or adverse events. This uncontrolled observational case series reports on the safety and feasibility of the use of micro-fragmented adipose tissue injections for the treatment of 10 consecutive female patients with symptomatic vaginal atrophy, vulvovaginal dystrophy, and/or stress incontinence.

\section{METHODS}

\section{Ethics}

The procedure described in this observational case series was performed in accordance with Good Clinical Practice (National Institute for Health Research [NIHR]) and the General Medical Council (GMC) guidelines on research, patient consent to research, and future publication. The Lipogems system used to harvest and process the adipose tissue was a U.S. Food and Drug Administration (FDA)-approved product for the use of a number of surgeries, inclusive of gynaecological surgeries.

\section{Patients}

Ten women with a multitude of conditions, including vaginal atrophy, vulvovaginal dystrophy, and SUI, agreed to treatment by injection of autologous micro-fragmented adipose tissue. These patients had previously undergone various therapies with unsatisfactory symptom relief and sought out an alternative treatment modality. Patients were consulted by a gynaecological consultant and an anaesthetic consultant, in which suitability for the procedure and anaesthesia were assessed. Clinical diagnoses were made upon vulvovaginal examination. A decision to perform biopsy was made on a case-by-case basis and was not performed on every woman due to the risk of fibrosis and impact on the fragility of tissue to be injected.

Consent forms were sent to patients 2-3 weeks in advance of their procedure; they were advised on the risks and benefits of the procedure and made aware of any alternative treatments available, in line with GMC guidance on consent. All patients completed an online preassessment questionnaire to assess their health, BMI, and past medical history, which is displayed along with gynaecological diagnoses in Table 1.

\section{Harvesting the Adipose Tissue}

Harvest entailed tumescent liposuction, which was undertaken by an experienced (consultant grade) plastic surgeon. The most commonly used harvest (donor) site was the lower anterior abdomen, performed with the patient in a supine position. However, in patients with a lower BMI, the harvest was carried out with the patient in prone position, with donor sites including lumbar flanks, superolateral buttocks, and/or lateral thighs. The tumescent fluid comprised $0.9 \%$ normal saline and adrenaline (concentration of 1:500,000). The tumescent fluid did not include local anaesthetic because these (lidocaine and ropivacaine) are reported to have proapoptotic influences on adipose derived stromovascular fraction. ${ }^{32}$

A manual (nonautomated) and gentle (low vacuum and low shear) tumescent liposuction technique was adopted. A minimum of 200 $\mathrm{mL}$ of lipoaspirate was harvested from each patient; in slender patients more was required. The final processed fraction yield equated to approximately $10 \%$ of lipoaspirate volume, and therefore, approximately $20 \mathrm{~mL}$ of processed fraction was typically available for the operating gynaecologist.

At completion of tumescent liposuction harvest, each donor site used was infiltrated with approximately $50 \mathrm{~mL}$ of normal saline $0.9 \%$, containing safe and appropriate concentrations of bupivacaine, tranexamic acid, and adrenaline. This step was employed to ameliorate postoperative donor site pain and bruising. Finally, donor sites were dressed with wound closure tape, simple gauze, and adhesive dressing, with application whenever possible (subject to fit) of a post-operative surgical compression band or garment.

\section{Processing of the Adipose Tissue}

The lipoaspirate was immediately processed, eliminating proinflammatory agents, such as oil from ruptured adipocytes and red blood cells, by filtering and washing. Sequential micro-fracturing ensured small and uniform particle size, which is subsequently important for smooth (low shear) injection. The trauma imparted to the cells during the micro-fracturing steps is important in promoting pericyte cell activation and MSC differentiation. 


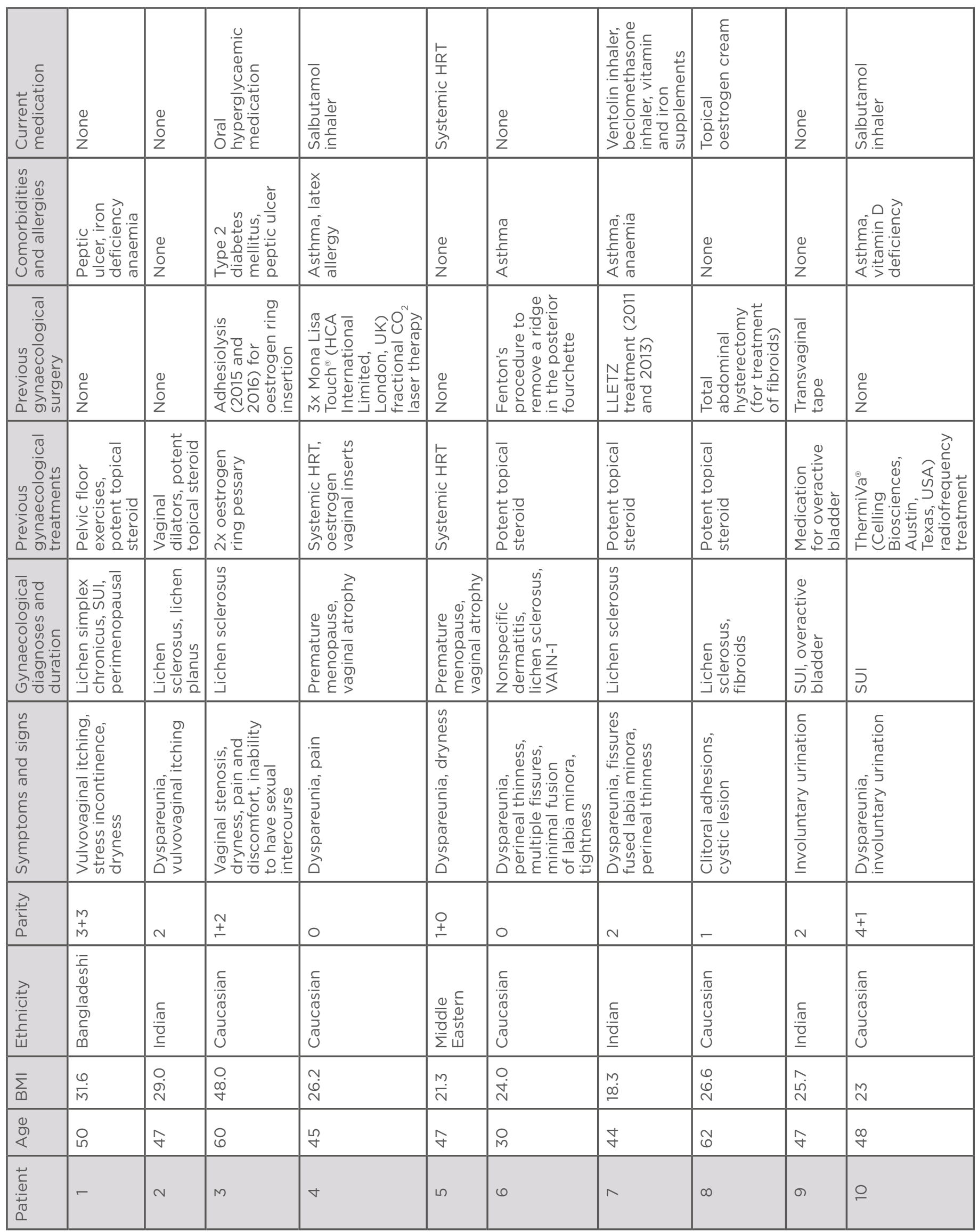


This trauma is imparted to cells in the context of total fluid (normal saline) immersion, which in turn dissipates energy and reduces cell rupture rates because cells need to be activated rather than ruptured. The final fraction was expressed from the device in $10 \mathrm{~mL}$ syringes and after settling for 5 minutes, saline subnatants were discarded. The fractions were then decanted into multiple $1 \mathrm{~mL}$ Luer-lock syringes in preparation for injection by the operating gynaecologist.

\section{Micro-Fragmented Adipose Tissue Injection}

The genitourinary area was inspected and scored using the vaginal health index $(\mathrm{VHI})$, and patients were placed in the lithotomy position for injection. If needed, the gynaecologist then performed a biopsy using small disposable punch biopsy forceps.

Micro-fragmented adipose tissue was injected using a 20 gauge blunt cannula in 0.5-1.0 $\mathrm{mL}$ aliquots into the subcutaneous and subepithelial vaginal space. This was performed by an experienced gynaecologist (consultant grade) in a homogeneous and uniform manner following a patient-specific topographic map, which indicated the areas of genitourinary changes that were causing problems for each individual patient. Patients who experienced stress incontinence had additional injections into paraurethral areas using a temporary 12 French unit Silastic ${ }^{\circledR}$ (C.R. Bard, Inc, New Providence, New Jersey, USA) urinary catheter to assist in identification of the urethral anatomy. An average of $24.0 \mathrm{~mL}$ microfragmented adipose tissue was injected per case, ranging from 8.5 to $32.0 \mathrm{~mL}$. Following the injections, unless contraindicated, a Voltarol $^{\circledR}$ (GlaxoSmithKline, London, UK) suppository was given to the patients with prior consent. Total time for harvesting and processing adipose tissue into micro-fragmented adipose was 20-30 minutes, and the gynaecological injection phase lasted 20-30 minutes. The total operation time was under 1 hour for every case.

\section{Recovery and Discharge}

Patients were discharged the same day in accordance with the guidelines by the British Day Surgery Association (BADS) which ensures all women can freely pass urine. Patients were discharged with a prescription of paracetamol $1 \mathrm{~g}$ once daily, dihydrocodeine $30 \mathrm{mg}$ once daily, lactulose $10 \mathrm{~mL}$ twice daily (to combat the constipating effects of dihydrocodeine only), and clotrimazole $1 \% \mathrm{w} / \mathrm{w}$ cream taken as needed in case of postoperative candidiasis. All women were provided with verbal and written advice regarding an abdominal binder to be worn, wearing abdominal dressings at the adipose harvest sites, and advice regarding time until resuming sexual activity, shaving, bathing, and heavy lifting.

\section{Outcome Measures}

All patients received a follow-up telephone consultation from a registered nurse 48 hours after their procedure to assess signs of infection, pain, dysuria, skin irregularities, skin discolouration, and discharge.

All patients received consultations with a consultant gynaecologist before the procedure, and at 2 weeks, 3 months, 6 months, and 1 year postoperation, as displayed in Figure 1. In addition to this, further consultations are planned for 2 years postoperation, or more frequently if required. These consultations involved a clinical examination, and outcomes were measured using the Female Sexual Function Index ([FSFI]: 2-36, in which 36 is a good result), the International Consultation on Incontinence Questionnaire-Urinary Incontinence Short Form ([ICIQ-UI SF]: O-21, in which 0 is a good result), the Vulvovaginal Symptoms Questionnaire ([VSQ]: 0-200, in which 200 is a good result), and the Short-Form 12 ([SF-12]: 10-70, in which 70 is a good result) health survey.

\section{RESULTS}

No intraoperative or postoperative adverse events or surgical complications were reported. During the recovery period, only one woman requested paracetamol for pain relief. The total time for this treatment as a day case from admission to discharge was 120-180 minutes per case. Postoperatively, no issues were reported relating to significant pain, discharge, signs of infection, or candidiasis. Mild donor site (tumescent liposuction) bruising, tenderness, and skin contours were in line with preoperative expectations and caused no significant concerns in any of the women in the cohort. 


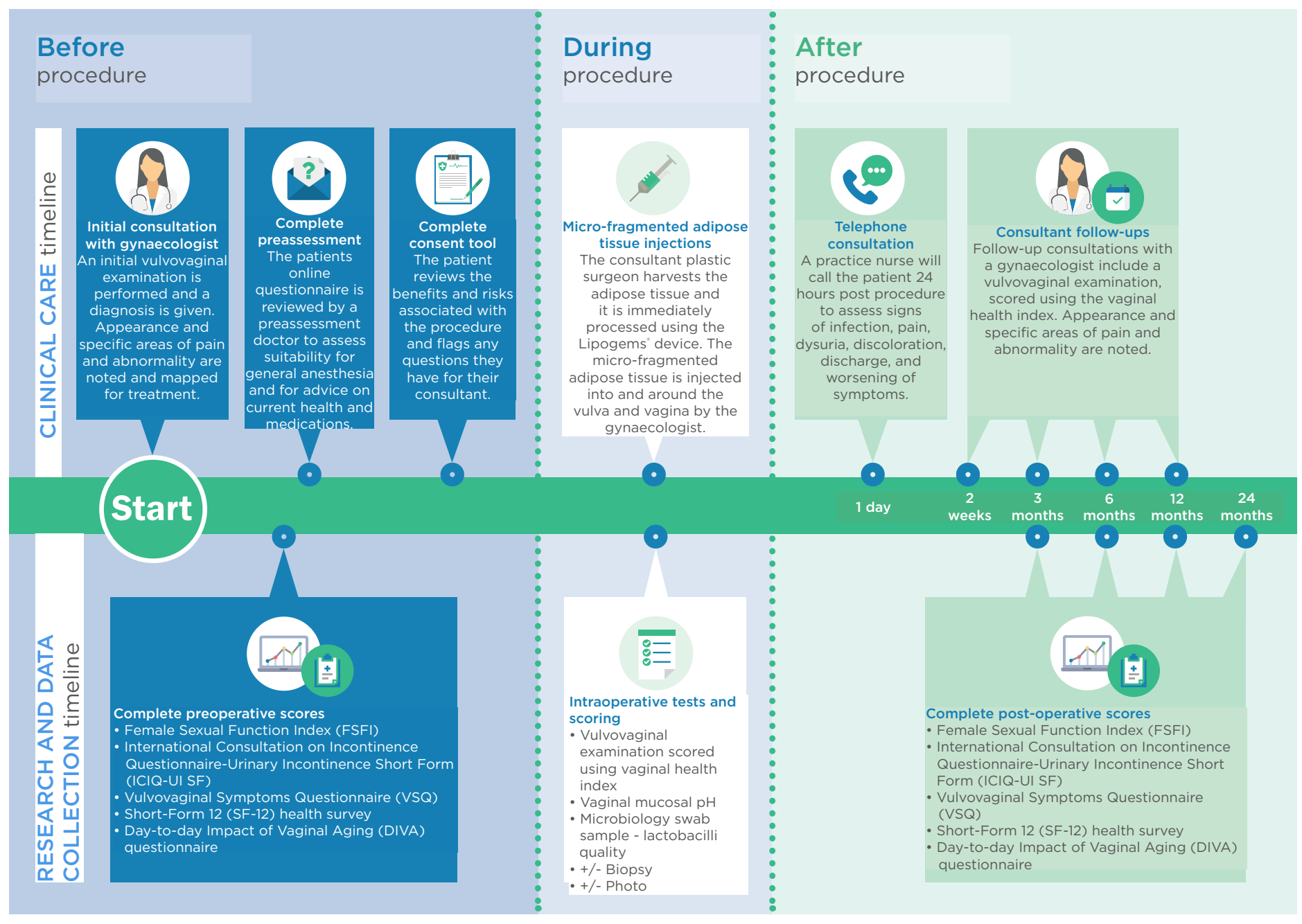

Figure 1: Follow-up and outcome measures for patients.

\section{Patient 1}

The patient presented with lichen simplex chronicus, vulvovaginal itching, SUI, and dryness, and upon examination there were signs of vaginal atrophy. The patient reported significant improvement of SUI and dryness at 6 months, with only occasional vulvovaginal itching. Examination revealed improvement of the appearance of the vulvar area with no vaginal atrophy. Unfortunately, this patient was not followed-up for scores.

\section{Patient 2}

The patient presented with lichen sclerosus with dyspareunia and vulvovaginal itching. The patient reported no dyspareunia at 3 and 6 months. At 16 months, the patient reported very mild dyspareunia, but complete resolution of vulvovaginal itching. A tender area of fibrosis was noted on the anterior vaginal wall on examination, but there was significant improvement in the appearance of the vulva, with no lichenification present. Overall, her scores had improved at 6 months: FSFI (21.5 to 29.1); VSQ (101 to 0); mental SF-12 (44 to 61). Her physical SF-12 decreased from 58 to 57. At 1 year, her scores demonstrated sustained improvement in her symptoms: FSFI (27); VSQ (14); mental SF-12 (56); and physical SF-12 (58).

\section{Patient 3}

The patient presented with lichen sclerosus and severe pain and discomfort, which precluded sexual intercourse. Vaginal examination was impossible due to stenosis and fibrosis; insertion of one finger was impossible due to pain. In the 6,12 , and 16 months following treatment, vaginal examination was possible with a small 
speculum and the patient was able to insert vaginal dilators herself. There was still tightness of the vaginal wall as a result of fibrosis, but her ring pessary was more easily changed, with no pain, and the urethra was visible, which had previously been hidden by adhesions. Scores had improved at 6 months: FSFI (10.2 to 13.2); ICIQ-UI (10 to 7); VSQ (56 to 38); and physical SF-12 (48 to 54). Mental SF-12 decreased (61 to 580 .

\section{Patient 4}

The patient presented with pain, dryness, and dyspareunia. Speculum examination previously caused severe pain and showed vaginal atrophy and dryness. There was complete resolution of dyspareunia and dryness at 3, 12, and 16 months, with only some occasional burning in the posterior fourchette. Speculum examination was performed without pain, and the vaginal wall appeared healthy and well lubricated. Scores had all improved at 6 months: FSFI (6.3 to 6.6); ICIQ-UI (4 to 0); VSQ (168 to 10); mental SF-12 (30 to 58); and physical SF-12 (34 to 41).

\section{Patient 5}

The patient presented with vaginal atrophy. Preoperative biopsy showed evidence of chronic inflammation, and the patient experienced dyspareunia and dryness. At 6-month follow-up this had improved significantly, and the patient reported a healthy discharge that was not present previously. This was also the case at 1 year and 16 months following treatment. Scores at 6 months and 1 year were the same: FSFI ( 5 to 20 ); VSQ (45 to 25 ); mental SF-12 (47 to 47 ); and physical SF-12 (29 to 29).

\section{Patient 6}

The patient presented with dyspareunia, chronic dermatitis, lichen sclerosus, and lowgrade vaginal intraepithelial neoplasia. On examination, there was significant lichenification with raw skin around the perineum and fusion of the labia minora. No pain in the perineal area was reported at 6 months, but the patient had not been sexually active so could not comment on dyspareunia. Mild discomfort was reported around the labia minora where an inflamed pimple was found, and antibiotics prescribed. Vulvovaginal examination showed significant improvement, with no fissures or raw skin. Some scores improved at 6 months: VSQ (145 to 67) and physical SF-12 (57 to 59). Some scores mildly worsened: FSFI (4.4 to 3.6) and mental SF-12 (51 to 39). However, FSFI was difficult to measure because the patient had not been sexually active.

\section{Patient 7}

The patient presented with severe lichen sclerosus, dyspareunia and fissures following sexual intercourse, vulvovaginal itching, and dryness. Examination revealed labia minora fusion, perineal thinness, and raw skin on the perineum. Complete resolution of vulvovaginal itching, dryness, and dyspareunia was reported at 6 weeks and 9 months following treatment. Examination revealed a significant improvement in the appearance of the vulvovaginal area, with no evidence of lichenification. The authors were unable to obtain scores from this patient at 6 months, but there was improvement at 3 months: FSFI (4.4 to 5.3), ICIQ-UI (3 to 0), VSQ (89 to 55), and physical SF-12 (53 to 57). However, her mental SF-12 mildly decreased (56 to 47).

\section{Patient 8}

The patient presented with lichen sclerosus. Complete resolution of dryness was reported at 4 months, as well as significant improvement in vulvovaginal itching, but the patient could not comment on her dyspareunia because she had not had sexual intercourse due to other factors. On examination, the vulvovaginal area appeared healthier, particularly the labia majora, but there were still some small patches of lichen sclerosus around the anus. The following scores improved at 6 months: FSFI (3.8 to 8.4), VSQ (110 to 103), and mental SF-12 (32 to 38). However, there was a minimal decrease in physical SF-12 (60 to 59).

\section{Patient 9}

The patient presented with severe SUI. There was complete resolution of SUI at 2 weeks, though some symptoms returned at 3 months. However, SUI symptoms had significantly improved from her pre-operative state. Unfortunately, this patient was lost to follow-up after 3 months and therefore her scores are not available. 


\section{Patient 10}

The patient presented with SUI and dyspareunia. Within 2 weeks the patient had noticed significant improvement of SUI, which was also reported at 1,2, and 3 months follow-up. Additionally, the patient reported increased pleasure with sexual intercourse with no dyspareunia. Her scores improved at 6 months: FSFI (39.0 to 33.3), ICIQ-UI (8 to 5), physical SF-12 (40 to 55 ), and mental SF-12 (59 to 60).

\section{DISCUSSION}

Vaginal atrophy, vulvovaginal dystrophies, and SUI are conditions that can have detrimental effects on a woman's urogenital health, sexual function, and psychological wellbeing. Whilst current approaches can relieve symptoms, they require ongoing use, which can increase the risk of nonadherence. Additionally, some of the first-line treatment approaches currently used are associated with risks and complications, and do not always completely resolve symptoms.

This observational case series demonstrates the safety and feasibility of autologous micro-fragmented adipose tissue injections in the treatment of 10 women with vaginal atrophy, vulvovaginal dystrophy, and SUI. It is the largest case series to date that uses this procedure to treat women with these conditions. To the best of the authors' knowledge, it is the first case series that demonstrates the safety and feasibility of the described procedure for the treatment of vulvovaginal dystrophies such as lichen sclerosus and lichen planus, as well as SUI. Results are consistent with previous case reports of vaginal atrophy by Fantasia et al. ${ }^{27}$ who treated one woman, and Casarotti et al. ${ }^{28}$ who treated three women. Demonstration of the safety of this procedure is important in presenting a feasible treatment option for women who have found previous treatment modalities unsuccessful in resolving their symptoms. Additionally, where local and systemic hormonal therapies are contraindicated (women with previous gynaecological or breast cancers), ${ }^{33}$ this treatment may act as a viable alternative.

The use of platelet-rich plasma (PRP) has been suggested for the treatment of the aforementioned conditions. ${ }^{31}$ Casabona et al. $^{26}$ used a combination of PRP and micro-fragmented adipose tissue injection for the treatment of 15 women diagnosed with lichen sclerosus and Aguilar et al. ${ }^{34}$ used a combination of PRP and hyaluronic acid, both with favourable outcomes. ${ }^{26}$ The advantage of the method described in this case series is that it is a simple, one-step method that can be performed intraoperatively, but the small sample size in both case series limits the ability for statistical analysis. Additionally, fractional $\mathrm{CO}_{2}$ laser therapy has been suggested as a treatment for vaginal atrophy. However, a comparative study has shown that autologous micro-fragmented adipose tissue injections are more effective in restoring healthy tissue, possibly due to the effect of trophic factors. ${ }^{35}$

For patients with vulvovaginal dystrophies such as lichen sclerosus and lichen planus, which are associated with vulvar malignancies, it is unknown whether early treatment lessens the risk of malignancy. Calcineurin inhibitors have been recently explored as a potential treatment, but there is concern surrounding the increased risk of potentiating malignant transformation due to their local immunosuppressive effects on tissue. ${ }^{36,37}$ In the future, it will be important to evaluate the effect that treatment with autologous micro-fragmented adipose tissue injections has on risk of malignant transformation of these conditions, and whether early treatment can further reduce this risk.

Preliminary results are promising and encourage further research into the efficacy of the treatment, with a long-term follow-up. It will be important to evaluate efficacy with objective measures such as vaginal $\mathrm{pH}$ and cytological studies. Whether such a technique may exert a synergistic effect with current treatment modalities such as systemic and local treatments remains to be fully investigated.

This case series is limited by the low number of participants, and the wide variety of complaints and diagnoses among the 10 patients. A limitation to the procedure is that a day surgery operating theatre is required, as well as the presence of a consultant gynaecologist, a consultant anaesthetist, and a plastic surgeon to carry out the highly specialised procedure. 


\section{References}

1. DiBonaventura $\mathrm{M}$ et al. The association between vulvovagina atrophy symptoms and quality of life among postmenopausal women in the United States and western Europe. J Womens Health (Larchmt). 2015;24(9):713-22.

2. Mac Bride MB et al. Vulvovaginal atrophy. Mayo Clin Proc. 2010;85(1):87-94.

3. Goldstein AT et al. Prevalence of vulvar lichen sclerosus in a general gynecology practice. J Reprod Med. 2005;50(7):477-80.

4. Coyne KS et al. The prevalence of lower urinary tract symptoms (LUTS) in the USA, the UK and Sweden: results from the epidemiology of LUTS (EpiLUTS) study. BJU Int. 2009;104(3):352-60.

5. Domoney $\mathrm{C}$ et al. The CLOSER survey: impact of postmenopausal vaginal discomfort on women and male partners in the UK. Menopause Int. 2013:19(2):69-76.

6. Palacios $\mathrm{S}$ et al. The European Vulvovaginal Epidemiological Survey (EVES): prevalence, symptoms and impact of vulvovaginal atrophy of menopause. Climacteric. 2018;21(3):286-91.

7. Nappi RE et al. Vulvar and vaginal atrophy in four European countries: evidence from the European REVIVE Survey. Climacteric. 2016;19(2):188-97.

8. Portman DJ, Gass MLS. Genitourinary syndrome of menopause: new terminology for vulvovaginal atrophy from the International Society for the Study of Women's Sexual Health and the North American Menopause Society. Menopause. 2014;21(10):1063 8.

9. Hill DA et al. Adherence to postmenopausal hormone therapy during the year after the initial prescription: a population-based study. Am J Obstet Gynecol. 2000;182(2):270-6.

10. Kingsberg SA et al. The Women's EMPOWER Survey: identifying women's perceptions on vulvar and vaginal atrophy and its treatment. $J$ Sex Med. 2017;14(3):413-24

11. Bleeker MCG et al. Lichen sclerosus: incidence and risk of vulvar squamous cell carcinoma. Cancer Epidemiol Biomarkers Prev. 2016;25(8):1224-30

12. Regauer $\mathrm{S}$ et al. Vulvar cancers in women with vulvar lichen planus: a clinicopathological study. J Am Acad Dermatol. 2014:71(4):698-707.

13. Cooper SM et al. Does treatment of vulvar lichen sclerosus influence its prognosis? JAMA Dermatol. 2004;140(6):702-6.

14. Neill SM et al. British Association of Dermatologists' guidelines for the management of lichen sclerosus 2010. $\mathrm{Br}$ J Dermatol. 2010;163(4):672-82.

15. Jonsson Funk $M$ et al. Long-term outcomes after stress urinary incontinence surgery. Obstet Gynecol. 2012;120(1):83-90.

16. Caplan AI, Dennis JE. Mesenchymal stem cells as trophic mediators. J Cell Biochem. 2006;98(5):1076-84

17. Gimble JM et al. Clinical and preclinical translation of cell-based therapies using adipose tissue-derived cells. Stem Cell Res Ther. 2010;1(2):19.

18. Zuk PA et al. Human adipose tissue is a source of multipotent stem cells. Mol Biol Cell. 2002;13(12):4279-95.

19. Bianchi $\mathrm{F}$ et al. A new nonenzymatic method and device to obtain a fat tissue derivative highly enriched in pericyte-like elements by mild mechanical forces from human lipoaspirates. Cell Transplant 2013;22(58):2063-77.

20. Garcia-Contreras M et al. Differences in exosome content of human adipose tissue processed by nonenzymatic and enzymatic methods. CellR4. 2014:3(1):e1423.

21. Dall'Oca C et al. Mesenchymal stem cells injection in hip osteoarthritis: preliminary results. Acta Biomed. 2019:90(Suppl 1):75-80.

22. Zeira $\mathrm{O}$ et al. Intra-articular administration of autologous microfragmented adipose tissue in dogs with spontaneous osteoarthritis: safety, feasibility, and clinical outcomes. Stem Cells Transl Med. 2018:7(11):819-28.

23. Pistoia V, Raffaghello L. Potential of mesenchymal stem cells for the therapy of autoimmune diseases. Expert Rev Clin Immunol. 2010;6(2):211-8.

24. Russo A et al. Autologous and micro-fragmented adipose tissue for the treatment of diffuse degenerative knee osteoarthritis. J Exp Orthop. 2017; doi:10.1186/s40634-017-0108-2.
25. Giuseppina Onesti $M$ et al. The use of human adipose-derived stem cells in the treatment of physiological and pathological vulvar dystrophies. Stem Cells Int. 2016;2016:1-6.

26. Casabona F et al. New surgical approach to lichen sclerosus of the vulva: the role of adipose-derived mesenchymal cells and platelet-rich plasma in tissue regeneration. Plast Reconstr Surg. 2010;126(4):210e-1e.

27. Fantasia $\mathrm{J}$ et al. Microfractured and purified adipose tissue ( Lipogems TM system ) injections for treatment of atrophic vaginitis. J Urol Res. 2016;3(7):1073.

28. Casarotti GA et al. Menopause: new frontiers in the treatment of urogenital atrophy. Eur Rev Med Pharmacol Sci. 2018;22(2):567-74.

29. Boero $\vee$ et al. Vulvar lichen sclerosus: a new regenerative approach through fat grafting. Gynecol Oncol. 2015;139(3):471-5.

30. Tamburino $\mathrm{S}$ et al. The role of nanofat grafting in vulvar lichen sclerosus: a preliminary report. Arch Plast Surg. 2016;43(1):93-5.

31. Kim SH et al. Rejuvenation using platelet-rich plasma and lipofilling for vaginal atrophy and lichen sclerosus. J Menopausal Med. 2017;23(1):63-8.

32. Goldman JJ et al. Tumescent liposuction without lidocaine. Plast Reconstr Surg Glob Open. 2016;4(8):e829.

33. Sturdee DW, Panay N Recommendations for the management of postmenopausal vaginal atrophy. Climacteric. 2010;13(6):509-22

34. Aguilar P et al. Novel technique of vulvo vaginal rejuvenation by lipofilling and injection of combined platelet rich plasma and hyaluronic acid: a case report. Springerplus. 2016:5(1):1184.

35. Azzam OA et al. Fractional $\mathrm{CO}(2)$ laser treatment VSQ autologous fat transfer in the treatment of acne scars: a comparative study. J Drugs Dermatol. 2013;12(1):e7-13.

36. Fischer G, Bradford J. Topica immunosuppressants, genital lichen sclerosus and the risk of squamous cell carcinoma: a case report. J Reprod Med. 2007;52(4):329-31.

37. McPherson T, Cooper S. Vulval lichen sclerosus and lichen planus. Dermatol Ther. 2010;23(5):523-32 